\title{
Relativistic electron beam propagation in the earth's atmosphere: modeling results
}

\author{
T. Neubert ${ }^{1,3}$, B. Gilchrist ${ }^{1}$, S. Wilderman ${ }^{2}$, L. Habash ${ }^{1}$, and H. J. Wang ${ }^{2}$
}

Abstract. Linear accelerators (linacs), capable of producing $5 \mathrm{MeV}$ energy electron beams at $80 \mathrm{~mA}$ currents, are now down to a size that allow them to be flown on sounding rockets or balloons. This opens up new opportunities for atmospheric/ionospheric modification experiments where the mesosphere and thermosphere regions of the atmosphere can be perturbed down to $40 \mathrm{~km}$ altitude. In this paper beam propagation and atmospheric perturbation effects are studied by Monte Carlo simulations and by analytical means. It is shown that the earth's magnetic field severely limits the radial expansion of the beam otherwise induced by electron-neutral collisions. It is also shown that the so-called "envelope-equations" from high-energy laboratory physirs adequately describe beam propagation in the upper atmosphere. The plasma density and electric conductivity modifications to the atmosphere are calculated from the Monte Carlo simulations. Inside the beam the conductivity in the $40-50 \mathrm{~km}$ altitude region is enhanced more than one order of magnitude by a $10 \mu \mathrm{s}$-duration pulse. Some ideas for future scientific investigations are discussed, including the generation of electrical discharges by bearns injected over thunderstorm regions.

\section{Introduction}

The dynamics of an MeV-energy electron beam injected into the atmosphere from ionospheric altitudes was studied by Banks et al. [1987] and Banks et al. [1990]. The motivation behind the study was the realization that linear accelerators $(5 \mathrm{MeV})$ were small enough in weight and size to be flown in space. While this early effort did not include magnetic field effects on beam propagation, the importance of magnetic field focusing effects was identified. The problem was simply stated: without a magnetic field, the beam undergoes radial expansion due to collision processes, which dramatically reduces the expected level of atmospheric perturbation.

Continuing the work by Banks et al. [1990], this paper presents models of beam propagation and atmo-

\footnotetext{
${ }^{1}$ Space Physics Research Laboratory, University of Michigan

${ }^{2}$ Department of Nuclear Engineering, University of Michigan

${ }^{3}$ Danish Meteorological Institute
}

Copyright 1996 by the American Geophysical Union.

Paper number 96GL00247

0094-8534/96/96GL-00247\$05.00 spheric modification where magnetic field effects are included. Two avenues are followed, (1) Monte Carlo simulations and (2) an analytical description by the so-called envelope equations [Humphries, 1990]. Both of these methods rely on tools that are developed for vastly different conditions from those found in the atmosphere. The Monte Carlo beam code is an out-growth of high-energy physics and medical applications, i.e., short distance beam propagation in dense materials, and the envelope equations are derived for the case of high-current beams ( $\mathrm{kA}$ ) in the laboratory. As we shall see, we have been successful in adapting both methods to atmospheric conditions and that the results show very good agreement. This means that in many cases one can rely on the much simpler analytical treatment of the problem, resorting to Monte Carlo simulations only in special cases where a more accurate description is needed.

The results presented here form the foundation for future studies of optical emission characteristics, modifications of the atmospheric electric potential and other aspects of relativistic beam dynamics.

\section{The Envelope Equations}

The envelope equations are closed-form analytical expressions describing the envelope of electron beams as they propagate in complex field geometries and undergo collisional interactions with ambient neutral gas and plasma [Humphries, 1990]

$$
\begin{gathered}
\frac{d^{2} R}{d z^{2}}=-\frac{d \gamma / d z}{\beta^{2} \gamma} \frac{d R}{d z}-\left[\frac{\omega_{c e}}{2 \beta \gamma c}\right]^{2} R \\
+\frac{\left\langle\theta^{2}\right\rangle}{R}+\left[\frac{R_{o}^{2} \omega_{c e}}{2 \beta \gamma c}\right]^{2} \frac{1}{R^{3}} \\
\frac{d\left\langle\theta^{2}\right\rangle}{d z}=\left.\frac{d\left\langle\theta^{2}\right\rangle}{d z}\right|_{c}+\frac{2 \gamma}{\gamma^{2}-1}\left\langle\theta^{2}\right\rangle \frac{d \gamma}{d z} \\
\left.\frac{d\left\langle\theta^{2}\right\rangle}{d z}\right|_{c}=\frac{16 \pi N Z(Z+1) r_{e}^{2}}{\gamma^{2} \beta^{4}} \ln \left[\frac{204}{Z^{0.333}}\right] \\
\frac{d \gamma}{d z \quad}=\frac{2 \pi N Z r_{e}^{2}}{\beta^{2}} \cdot\left[\ln \left(\frac{1}{2}\left(\frac{E^{o}}{I}\right)^{2} \beta^{2} \gamma^{2}(\gamma-1)\right)\right. \\
\left.-\ln ^{2}\left(\frac{2}{\gamma}-1+\beta^{2}\right)+\frac{1}{\gamma^{2}}+\frac{(1-1 / \gamma)^{2}}{8}\right]
\end{gathered}
$$

The beam is propagating in the $z$-direction, and the radius of the beam, $R$, is a function of $z$. These are the envelope equations per se, where we have only retained terms that are of significance for the problem at hand. In (1), the first term on the right-hand side rep- 
resents focusing processes arising from acceleration or deceleration of electrons with position along the $z$-axis. The second term represents applied magnetic fields, in our case the earth's magnetic field, while the third term describes defocusing processes from scattering and deceleration. The final term represents the defocusing effect arising from the conservation of canonical angular momentum. In the equations, $R_{o}$ is the beam radius at beam injection, $\gamma$ is the relativistic factor, $\beta$ is $v / c$, $c$ is the velocity of light, and $\omega_{c e}$ the non-relativistic electron gyro frequency.

Equation (2) describes defocusing by emittance due to collisions (first term) and deceleration (second term). Here $\left\langle\theta^{2}\right\rangle$ is the mean-squared divergence angle. The small-angle scattering formula is shown in (3), where $N$ and $Z$ are properties of the medium, $r_{e}$ is the Bohr radius, and $\gamma$ and $\beta$ are functions of $z$. The equations are closed by the Bethe formula (4), which gives the collisional stopping power for relativistic electrons. Here $I$ is the mean ionization potential and $E^{o}$ the electron rest energy $m_{e} c^{2}$.

\section{Monte Carlo Simulations}

Two distinct transport algorithms have been applied. In one instance, the EGS4 program, which has been used extensively and thoroughly benchmarked by both the high energy physics and medical physics communities, was employed [Nelson et al., 1985]. EGS4 uses the condensed random walk transport algorithm in which a particle's full transport path is broken into roughly a hundred segments. Aggregate deflection and ionization through each segment is assumed to be described by analytically derived distribution functions, and the full particle path is modeled by successive application of appropriate segment-wise scattering distribution data. As a means of verification, a second more computer intensive program, which treats all elastic scattering events individually and employs best available differential scattering distribution data, was also applied to this problem. Both simulations produce similar results.

The atmosphere is modeled as eleven separate $2 \mathrm{~km}$ thick "slabs" composed of $70 \% \mathrm{~N}_{2}$ and $30 \% \mathrm{O}_{2}$, with constant local density. Energy deposition is tallied for cubic regions of volume $(1 \mathrm{~km} \mathrm{x} 1 \mathrm{~km} \mathrm{x} 100 \mathrm{~m})$ for simulations with no magnetic field and $(100 \mathrm{~m})^{3}$ for simulations which include a magnetic field. The deposition profiles in depth and in the lateral dimensions are determined in this way.

\section{Results}

In the Monte Carlo simulations, a $5 \mathrm{MeV}$-beam is injected downwards from $60 \mathrm{~km}$ altitude. We chose 60 $\mathrm{km}$ to limit the computational effort. The atmosphere above this altitude is relatively tenuous, and moderate scattering/ionization is experienced above $60 \mathrm{~km}$.

The atmosphere is the MSIS86 model for night-time, mid-latitude conditions [Hedin, 1987]. Figure 1 shows the fractional energy deposition as a function of altitude. The black curve represents the Monte Carlo re- sults, and the red curve results obtained using the Bethe formula. The very good agreement between the two was anticipated since, after all, the EGS4 code incorporates the Bethe formula.

The radial expansion of the beam without an ambient magnetic field is shown in Figure 2. The fractional energy deposition as a function of altitude and horizontal distance is shown on a gray-scale together with the analytical results obtained from the envelope equations. We notice an excellent agreement between the two approaches. It is also evident that the beam experiences substantial spreading in the horizontal direction, up to $8 \mathrm{~km}$ at $42 \mathrm{~km}$ altitude, caused by diffusion induced by electron-neutral scattering.

The question is now to what extent the magnetic field contains the radial beam spreading, and thereby focus and enhance the perturbation of the atmosphere. Results of a Monte Carlo simulation including a vertical magnetic field of 0.4 Gauss is shown in Figure 3. The beam is now well contained, and the beam radius is within $400 \mathrm{~m}$, which is less than the $607 \mathrm{~m}$ gyro radius of a $5 \mathrm{MeV}$-beam at $90^{\circ}$ pitch angle (notice the change of horizontal scale). The analytical results are also in overall agreement but they contain oscillations associated with the gyromotion of the electrons. These oscillations have a wavelength $\lambda_{c}$ determined by the magnetic field strength and the characteristic beam energy parallel to $B$ according to $\lambda_{c}=2 \pi v_{\|} \gamma / \omega_{c e}$. Thus $\lambda_{c}$ decreases with altitude as the beam electrons lose energy and obtain larger pitch angles. Similar results were found in Nauryzbaev [1988]. The Monte Carlo simulation is the combined result of both primary beam electron and secondary electron energy deposition rates and do not, therefore, contain the same oscillatory feature.

Electron Density. The electron density can be estimated from the fractional energy deposition by assuming one electron-ion pair created for every $35 \mathrm{eV}$ energy loss. The results are shown in Figure 4 . The beam parameters are those proposed for a sounding rocket experiment funded by U.S. Air Force [Jost, 1993], where the beam energy is $5 \mathrm{MeV}$, the beam current $80 \mathrm{~mA}$, and the pulse duration $10 \mu \mathrm{s}$. It is assumed that recombination occur on a longer time-scale $(\mathrm{ms})$, so the densities shown are those immediately after one beam pulse injection.

Densities are of the same order of magnitude as ambient ion densities, mostly created by cosmic radiation. Higher densities can be achieved by repeated pulse injections. In this case, the effects of the rocket velocity across the magnetic field, recombination rates, and space-charge field acceleration of charged particles must be taken into account.

Electric Conductivity. The atmospheric electric conductivity resulting from the beam injection is shown in Figure 5 . The background conductivity increases with altitude according to Volland [1984], primarily due to the presence of negative ions. The conductivity inside the beam is calculated from the information on the enhanced plasma density. It reaches values 1-2 orders of magnitude above the ambient conductivity. It is expected, therefore, that significant modification of the 


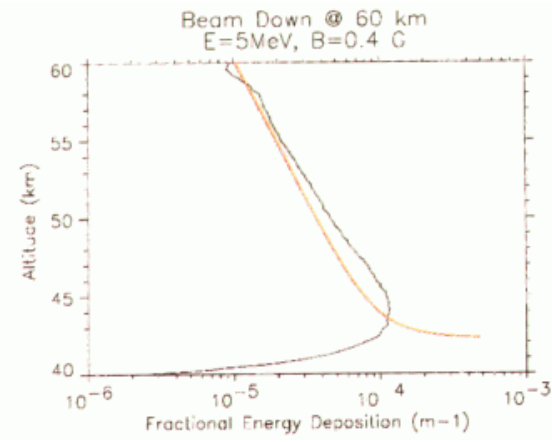

Figure 1. Fractional energy deposition vs. altitude. Red curve is the Bethe formula, and black curve the Monte Carlo simulation.

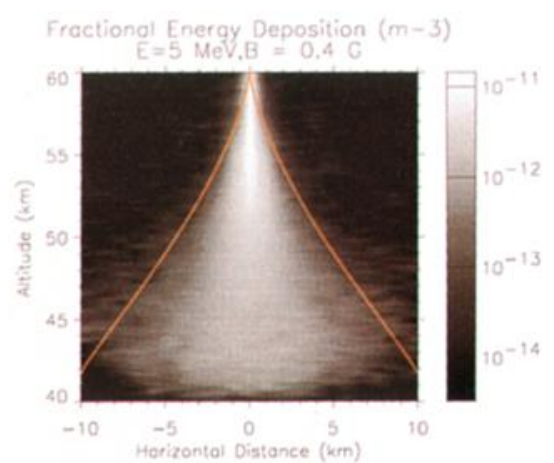

Figure 2. Beam cross-section of fractional energy deposition as a function of beam radius and altitude. The earth's magnetic field is not included. The simulations are shown on a grayscale and the solution of the envelope equations with the red curve.

ambient electric potential will be induced in and around the beam. A time-dependent calculation is needed to determine such effects because of the short exposure and perturbation time of a beam column.

\section{Discussion}

The study presented here suggests several new experiments. Perhaps the most spectacular is the modification of the atmospheric electric potential structure to allow discharges between the beam column and the ambient atmosphere. The recent reports of optical flashes above thunderstorms [Sentman et al., 1995; Wescott et

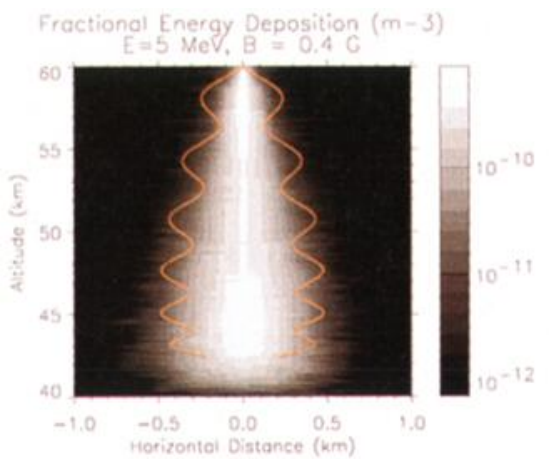

Figure 3. Same as Fig. 2, including the earth's magnetic field.

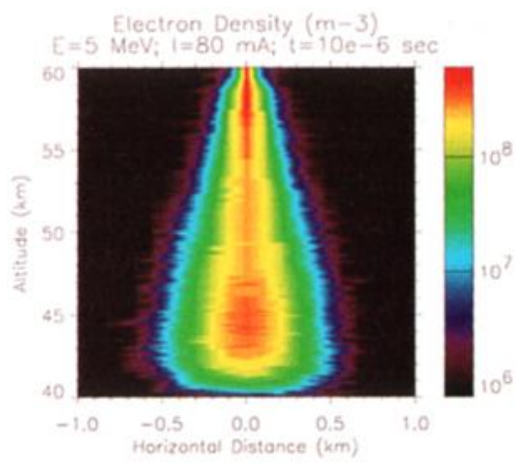

Figure 4. Electron density.

$a l, 1995]$, indicate that on occasion, discharges occur naturally above thunderstorms. A relativistic electron beam experiment may provide a new means of actively probing upper atmospheric electric phenomena.

To explore this idea further, we calculate the collumn resistance per unit length, $r$, from an estimate in Banks et al. [1990]

$$
r \simeq \frac{m_{e} \nu_{e n}}{n_{e} e^{2} A}
$$

where $\nu_{e n}$ is the electron-neutral collision frequency, $n_{e}$ the electron density, and $A$ the beam cross section area. Both the initial electron density in the column and the electron-neutral collision frequency are proportional to the neutral gas density so that $r$ is roughly constant with altitude. Using $50 \mathrm{~km}$ as a reference altitude we have $\nu_{e n} \simeq 10^{8} \mathrm{~s}^{-1}$, and from Figure 4 that $n_{e} \simeq 10^{8}$ $\mathrm{m}^{-3}$ and $A \simeq 10^{5} \mathrm{~m}^{2}$, which gives $r \simeq 360 \Omega / \mathrm{m}$.

The resistance in the column is therefore substancial, and a new electric potential distribution will be established with a time constant determined by the diffusion time of the electric field into the column. This time constant, $\tau_{c}$, was estimated in Banks et al. [1990]

$$
\tau_{c} \simeq \frac{\nu_{e n}}{\omega_{p e}^{2}}
$$

Using the values for electron density from Figure 4, we find the time constant at $50 \mathrm{~km}$ altitude to be $\tau_{c} \simeq 300 \mu \mathrm{s}$. It is of interest to compare the diffusion time of the electric field with the life-time of the ionization enhancement. Electrons are most rapidly lost through attachment to $\mathrm{O}_{2}$. The electron-negative ion equilibrium time constant is $\simeq 23 \mathrm{~ms}$ at $50 \mathrm{~km}$ altitude

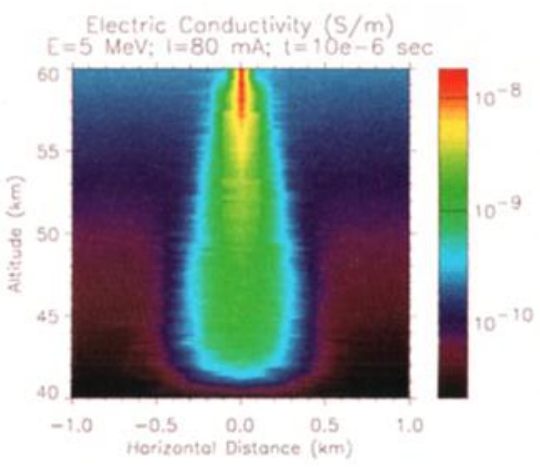

Figure 5. Electric conductivity. 
[Banks et al., 1990]. The life-time of the electron density enhancement in the beam column is therefore sufficient for the electric field to diffuse into the beam and thereby modify the electric potential structure around the beam.

The average potential difference between ground and the ionosphere is about $300 \mathrm{kV}$, with the ionosphere positive and ground negative. Most of the potential drop occurs over the lowest few km altitude where the neutral density is high and the conductivity low. Under fair weather conditions, the electric fields above 50 $\mathrm{km}$ altitude are of the order of $0.1 \mathrm{~V} / \mathrm{m}$ or smaller with a total potential increase from $50 \mathrm{~km}$ altitude to the ionosphere of $\simeq 1 \mathrm{kV}$. The potential between the beam and the ambient atmosphere outside of the beam could then reach $1 \mathrm{kV}$ at $50 \mathrm{~km}$ altitude. With a beam radius at this altitude of $200 \mathrm{~m}$, this corresponds to an average electric field strength of $50 \mathrm{~V} / \mathrm{m}$. In comparison, the break-down electric field for discharge in the atmosphere varies from about $10 \mathrm{~V} / \mathrm{m}$ at $80 \mathrm{~km}$ altitude to $10 \mathrm{kV} / \mathrm{m}$ at $50 \mathrm{~km}$ altitude. It is possible, therefore, that at altitudes around $70 \mathrm{~km}$ and above discharges around the beam may be triggered. The study of these will provide new insights into the electrification of the earth -atmosphere -ionosphere system.

It may also be of interest to inject beams from the ionosphere and downwards over thunderstorm regions to directly probe the electric generator of the earthionosphere electric field. In this case the polarity is reversed and locations above cloud tops are positive with respect to the ionosphere. Averaged potential differences between cloud tops and the ionosphere are about $1 \mathrm{kV}$, and peak potentials are likely to be an order of magnitude larger or more. It is possible, therefore, that $\mathrm{MeV}$ beams injected over thunderstorms can trigger upward discharges similar to those observed occuring naturally. However, beam energies exceeding $5 \mathrm{MeV}$ may be needed. A model of the time dependent establishment of the electric potential in and around the beam is needed to estimate the beam-system parameters for triggering of upward lightning.

Other applications include the modification of chemical reaction paths in the middle atmosphere stimulated by naturally precipitating relativistic electrons. Particle precipitation can significantly affect the chemical composition by enhancing ionization and disassociation of atmospheric chemical composition as well as constituent temperatures. Transport winds and eddy mixing can extend these effects to regions far from those directly affected by the precipitation. Of particular interest are the production of odd-nitrogen and odd-oxygen species, since both lead to depletion of ozone in the stratosphere and mesosphere. It is possible that the active stimulation of the atmosphere by a powerful beam injector to- gether with optical spectroscopic detection techniques can be used for the study of these processes. Experiments as well as further modeling efforts are clearly needed to explore the potential of linacs in atmospheric research.

Acknowledgments. We are grateful for the valuable discussions held with Professors Y. Y. Lau and Peter M. Banks. The work was supported by AirForce contract F19628-93-k-003.

\section{References}

Banks, P. M., A. C. Fraser-Smith, B. E. Gilchrist, K. J. Harker, L. R. O. Storey, and P. R. Williamson, New concepts in ionospheric modification, $A F G L-T R-88-0133$, Air Force Geophysics Laboratory, 1987.

Banks, P. M., A. C. Fraser-Smith, and B. E. Gilchrist, Ionospheric modification using relativistic electron beams, AGARD Conference Proceedings, No 485, pp22-1, 1990.

Hedin, A. E., MSIS-86 thermospheric model, J. Geophys. Res., 92, 4649, 1987.

Humphries, Jr., S., Charged Particle Beams, WileyInterscience, 1990.

Jost, J., Spaceborne relativistic electron accelerator system, Final Report, SBIR program, Topic Number AF92-084, 1993.

Nauryzbaev, A. E., Dynamics of a relativistic electron beam in an external magnetic field, Soviet Physics - Lebedev Institute Reports, Allerton Press, New York, 1988.

Nelson, W. R., H. Hirayama, and D. W. O. Rogers, The EGS4 code system, SLAC Report-265, Stanford Linear Accelerator Center, 1985.

Sentman, D. D., E. M. Wescott, D. L. Osborne, D. L. Hampton, and M. J. Heavner, Preliminary results from the Sprites94 aircraft campaign: 1. Red sprites, Geophys. Res. Lett., 22, 1205, 1995.

Volland, H., Atmospheric Electrodynamics, Springer Verlag, 1984.

Wescott, E. M., D. Sentman, D. Osborne, D. Hampton, and M. Heavner, Preliminary results from the Sprites94 aircraft campaign: 2. Blue jets, Geophys. Res. Lett., 22, 1209, 1995 .

Brian Gilchrist and Linda Habash, Space Physics Research Laboratory, The University of Michigan, Ann Arbor, MI 48109

Torsten Neubert, Danish Meteorological Institute, Lyngbyvej 100, Copenhagen $\varnothing$, Denmark

Scott Wilderman and Hong Juan Wang, Department of Nuclear Engineering, The University of Michigan, Ann Arbor, MI 48109

(received September 6, 1995; revised December 18, 1995; accepted December 18, 1995.) 